\title{
Deformed octagon-hexagon-square structure of group-IV and group-V elements and III-V compounds
}

\author{
T. Gorkan, ${ }^{1}$ E. Aktürk $\odot,{ }^{1,2, *}$ and S. Ciraci ${ }^{3, \dagger}$ \\ ${ }^{1}$ Department of Physics, Adnan Menderes University, Aydin 09100, Turkey \\ ${ }^{2}$ Nanotechnology Application and Research Center, Adnan Menderes University, Aydin 09100, Turkey \\ ${ }^{3}$ Department of Physics, Bilkent University, Ankara 06800, Turkey
}

(Received 8 July 2019; published 19 September 2019)

\begin{abstract}
We report the prediction of a two-dimensional (2D) allotrope common to group-IV and group-V elements and III-V compounds, which consist of two nonplanar atomic layers connected by vertical bonds and form deformed octagon, hexagon, and squares (dohs) with threefold and fourfold coordinated atoms. Specifically for silicon, it is a semiconductor with cohesion stronger than silicene and can be chemically doped to have localized donor and acceptor states in the band gap. This allotrope can be functionalized to construct quasi-2D clathrates with transition metal atoms and attain spin polarized metallic, half-metallic, or semiconducting states. It is demonstrated that these properties can be maintained, when it is grown on a specific substrate. Stringent tests show that the atomic structure is dynamically stable and can sustain thermal excitation at high temperatures. Additionally, stable bilayer, as well as 3D layeredlike structures, can be constructed by the vertical stacking of single-layer dohs. Surprisingly, C, Ge, AlP, and GaAs can form also similar 2D semiconducting structures. In contrast to semiconducting black and blue phosphorene, $\mathrm{P}-d o h s$ is a semimetal with band inversion. While the premise of using well-developed silicon technology in 2D electronics has been hampered by the semimetallic silicene, the realization of this $2 \mathrm{D}$, semiconducting allotrope of silicon and compounds can constitute a productive direction in 2D nanoelectronics/spintronics.
\end{abstract}

DOI: $10.1103 /$ PhysRevB.100.125306

\section{INTRODUCTION}

The synthesis of free-standing and supported monolayers have brought new directions to explore exceptional material properties, primarily in two-dimensional (2D) electronics, photonics, and spintronics allowing high-density device packing and high-speed operation with low energy consumption. Graphene [1], the most renowned monolayer of the carbon atom, displays excellent mechanical properties and chemical inertness, but it is a semimetal with linear bands crossing at the Fermi level showing massless Dirac fermion and ambipolar characters, etc. However, the absence of an electronic energy band gap hindered graphene from many electronic applications. With the hope of exporting well-developed semiconductor device technology to two-dimensional (2D) materials and replenishing expectations, the monolayers of group-IV elements have been placed at the forefront of research on 2D materials. Motivated with the synthesis of graphene, elements and compounds like $\mathrm{Si}, \mathrm{GaAs}, \mathrm{GaN}$, etc., have been predicted to form monolayers in a buckled honeycomb structure [2]. Later, in an extensive study, the stability of the monolayers of silicon and germanium called silicene and germanene, respectively, have been demonstrated and their semimetallic electronic structures with linearly crossing bands at the Fermi level has been revealed [3]. Later, metallic and semiconducting nanoribbons of silicene depending on their chirality

\footnotetext{
*ethem.akturk@adu.edu.tr

†ciraci@fen.bilkent.edu.tr
}

have been also deduced [4]. Unusual properties of silicene and germanene have been extended to binary compounds of group-IV elements [5-7], and group III-V [7] and II-VI compounds [8] unveiling more than 20 monolayer structures. Among them, silicene was synthesized on $\operatorname{Ag}(111)$ surface [9-11] despite the objections that the 2D monolayer structure cannot be realized, since $\mathrm{Si}$ does not have a layered structure like graphite. Furthermore, the $\sqrt{3} \times \sqrt{3}$ pattern of silicene on $\operatorname{Ag}(111)$ has been unraveled [12]. The growth of multilayer silicene up to $\sim 100$ layers [13] has led to an important question of whether $\mathrm{Si}$ can have a layered structure like graphene $[14,15]$. Stable trigonal and honeycomb dumbbell structures, which were deduced to understand grown silicene $[12,16,17]$, initiated different types of silicon single-layers [18-24]. A similar dumbbell patterned monolayer has been predicted also for germanene [16]. The prime objectives of these recent theoretical studies have been to reveal 2D silicon based structures with high stability, high mobility, and sizable band gap for future nanodevice applications. Yaokawa et al. [21] realized the transformation of calcium intercalated silicene to a 2D silicene bilayer constructed four-, five-, and six-membered rings with an estimated band gap of $1.08 \mathrm{eV}$. Using a general global optimization method, Luo et al. [23] was able to deduce a Si bilayer consisting of seven- and five-membered rings having relatively lower energy. The dumbbell structure of $\mathrm{Si}$ is further exploited by Borlido et al. [19] to predict a low-energy, 2D semiconducting structure of Si by combining the silicene honeycomb structure with the dumbbell atoms arranged in a zigzag pattern. In another study [20], unprecedented 2D ultrathin Si structures 
were generated by $a b$ initio materials searching study with electronic properties ranging from semimetal to semiconductor. These structures had perfectly planar outermost surface made by distorted hexagonal rings and thickness depending on the arrangements of tetrahedron between these surfaces. Most recently, Zhuo et al. [18] claimed that they revealed 11 different 2D allotrope of Si based on the first-principles calculations on the structures designed by mixing threefold and fourfold coordinated $\mathrm{Si}$ atoms. These 2D materials were constructed from six-membered, eight- and four-membered, or eight- and five-membered rings combined with dumbbells and were predicted to be semiconducting behavior with high electron and hole carrier mobility. It appears that 2D singlelayer Si has several metallic, semimetallic, and semiconducting allotropes like 3D Si [25]. Even if the premise of using the silicon technology in 2D electronics has been hampered to great extent by the semimetallic state of silicene, presently, synthesizing a semiconducting, 2D single-layer or ultrathin $\mathrm{Si}$ and realizing a graphitelike, layered phase of silicon continue to be real challenges.

Based on state-of-the art first-principles calculations within the density functional theory (DFT), we predict that a $2 \mathrm{D}$ allotrope of $\mathrm{Si}$ is dynamically and thermally stable. It has fourfold and threefold coordinated Si atoms arranged in two nonplanar, atomic layers connected by vertical bonds [26] and consists of deformed octagons, hexagons, and squares (dohs). Surprisingly, it has cohesion stronger than silicene and an indirect band gap of $0.5 \mathrm{eV}$. When stacked vertically, this allotrope can also construct stable, metallic bilayers, as well as three-dimensional (3D) layeredlike structures. We name this allotrope of $\mathrm{Si} \mathrm{Si}$-dohs and propose also a possible kinetic path towards the realization of this 2D allotrope of Si. Even more surprising is that not only $\mathrm{Si}$, but also group-IV elements, like $\mathrm{C}, \mathrm{Ge}, \mathrm{III}-\mathrm{V}$ compounds like AlP, GaAs, even a group-V element, $\mathrm{P}$ can form stable dohs structures making a class of 2D materials.

\section{METHODOLOGY}

We have performed first-principles calculations for total energy and electronic structure within DFT by using planewave basis set and projector augmented wave (PAW) potential[27] as implemented in the Vienna Ab-initio Simulation Package, VASP $[28,29]$. The exchange-correlation potentials are represented by the generalized gradient approximation (GGA) with the Perdew-Burke-Ernzerhof (PBE) [30] exchange correlation functional including van der Waals ( $\mathrm{vdW}$ ) correction in the DFT-D2 level [31]. The kinetic-energy cutoff for the plane-wave basis set is taken as $500 \mathrm{eV}$ for $\mathrm{Si}$ and AlP, $550 \mathrm{eV}$ for $\mathrm{C}$, and $350 \mathrm{eV}$ for Ge and GaAs. Using the conjugate-gradient algorithm, all the atomic positions and lattice parameters are fully optimized until the forces on each atom are less than $1.0 \times 10^{-4} \mathrm{eV} / \AA$. The convergence criterion of self-consistent calculations is taken to be $10^{-6} \mathrm{eV}$ for total-energy values. Pressures on the lattice unit cell are reduced to values less than $1.0 \mathrm{kbar}$. Since the band gaps are underestimated by DFT using PBE, we also performed calculation using the Heyd-Scuseria-Ernzerhof (HSE06) hybrid functional method [32]. The screening length of HSE06 is taken as $0.2 \AA$, and the mixing rate of Hartree-Fock (HF) exchange potential is set to 0.25 .

The partial occupancies are determined using the Gaussian scheme with the smearing width of $0.01 \mathrm{eV}$. The cohesive energy (per atom) of Si-dohs is calculated by using the expression $E_{c}=E[\mathrm{Si}]-E[\mathrm{Si}-d o h s] / n$ in terms of the total energy of free $\mathrm{Si}$ atom and that of Si-dohs including $n \mathrm{Si}$ atoms in the unit cell. $E_{c}>0$ corresponds to the binding structure. Cohesive energies of compounds are calculated similarly by considering anion and cation energies and by averaging the calculated values to express them in eV per atom. Phonon dispersion spectra are computed using force constants of the supercell within the framework of density functional perturbation theory (DFPT) as implemented in the VASP code combined with the PHONOPY package [33] using an improved energy convergence criterion as $10^{-8} \mathrm{eV}$. These calculations were performed with a $3 \times 3 \times 1$ supercell and $6 \times 6 \times 1$ grid for the $k$-point for $\mathrm{Si}$-dohs. All ab initio molecular dynamics (AIMD) calculations were performed with a $2 \times 2 \times 1$ supercell and $k$-point grids are chosen to be $5 \times 5 \times 1$ for single and bilayers. A Nosé thermostat was used, and Newton's equation of motion were integrated through the Verlet algorithm with time steps of $1 \mathrm{fs}$. For bulk structure we choose $2 \times 2 \times 2$ supercells and $3 \times 3 \times 2 k$-point grids.

\section{SEMICONDUCTING, 2D-dohs STRUCTURE OF SI}

Our study starts with the open-framework, 3D allotrope of silicon structure $\mathrm{P} 6 / \mathrm{m}-\mathrm{Si}_{6}$ derived from $\mathrm{P} 6 / \mathrm{m}-\mathrm{NaSi}_{6}$ clathrates found by Sung et al. [34] as described in Fig. 1(a).
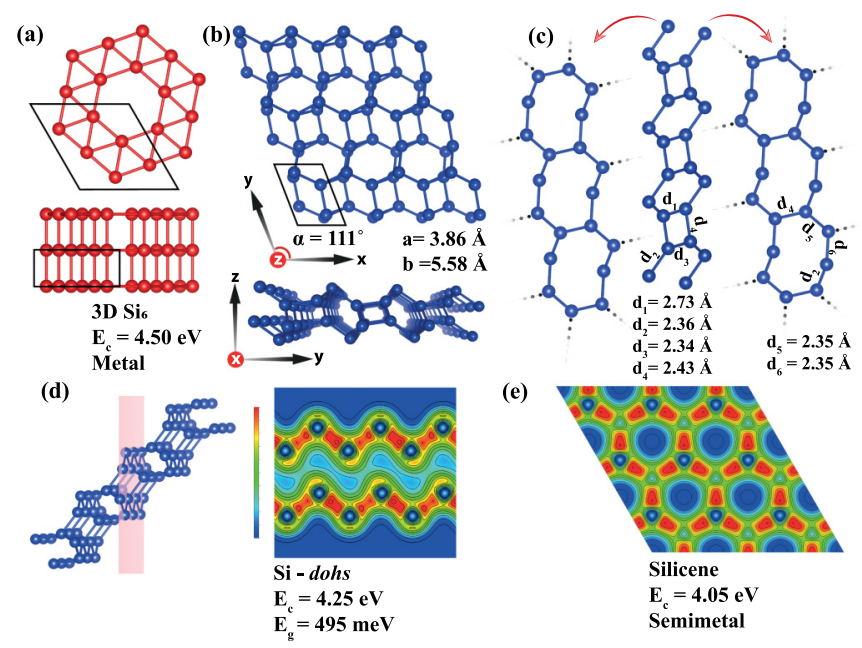

FIG. 1. (a) Top [(xy) plane] and side views of the metallic $\mathrm{P} 6 / \mathrm{m}-\mathrm{Si}_{6}$ clathrate structure with the calculated cohesive energy of $E_{c}=4.50 \mathrm{eV} /$ atom. (b) Top view of Si-dohs with the 2D oblique unit cell delineated. 2D lattice constants $\mathbf{a}$ and $\mathbf{b}$ are indicated. Its perspective side view below consists of deformed hexagons and squares. (c) Two nonplanar atomic layers of Si-dohs are made of deformed octagons, which are connected by two vertical bonds per unit cell and constitute deformed squares and hexagons in the side view. (d) Calculated charge density contour plots on a plane passing through specific Si-Si bonds described on the left. (e) Calculated charge density of semimetallic silicene monolayer is shown for comparison. 
Based on combining density functional calculation and the global optimization method of conformational space annealing, Sung et al. [34] reported that $\mathrm{P} 6 / \mathrm{m}-\mathrm{Si}_{6}$ and $\mathrm{P} 6 / \mathrm{m}-\mathrm{NaSi}_{6}$ structures are stable and exhibit the superconducting behavior at ambient pressure. It is a metallic phase of Si [35-38] and has a hexagonal lattice, where each $\mathrm{Si}$ atoms is sevenfold coordinated with $\mathrm{Si}$-Si bonds longer than the bond of the cubic diamond structure $(\mathrm{Si}-\mathrm{CD})$. This structure can be viewed as a stack of the atomic planes of nested double hexagons formed by fivefold coordinated, in-plane $\mathrm{Si}$ atoms, which are further connected to the adjacent atomic planes at the top and bottom site by vertical bonds. When cleaved from the stack, the free-standing atomic plane, which is very similar to the recently deduced [39] borophene phase named $\omega-\mathrm{B}$, is prone to the instability; eventually it transforms to the $2 \mathrm{D}$ Si-dohs structure shown in Figs. 1(b) and 1(c). The same structure is obtained also in the optimization of the $4 \times 4$ supercell of the atomic plane of $\mathrm{P} 6 / \mathrm{m}-\mathrm{Si}_{6}$. In the top view (i.e., in the $x y$ plane), Si-dohs is formed by deformed and buckled octagons; but in the side view it is made of deformed squares and hexagons. In the $2 \mathrm{D}$ oblique unit cell, two $\mathrm{Si}$ atoms are fourfold coordinated with bonds deviating from tetrahedral coordination; the remaining four $\mathrm{Si}$ atoms are nonplanar threefold coordinated. The bond lengths of fourfold coordinated atoms are comparable to those of CD structure. The distributions of electronic charge between nearest $\mathrm{Si}$ atoms are reminiscent of those of CD-Si. The cohesive energy is calculated to be $4.25 \mathrm{eV} /$ atom, which is $200 \mathrm{meV}$ higher than that of silicene.

Even if 2D Si-dohs appears to be a bilayer, it is not, for the following reasons: (i) The surfaces of atoms at both sides do not constitute stable monolayers; they are stabilized by vertical bonds [26] between them. (ii) Si-dohs is not constructed by the stacking of these two layers; it rather forms as a monolayer. The atomic structure also differs dramatically from those of silicene bilayers [40-42]. The question of whether this allotrope is stable is answered by the dynamical and thermal stability analysis as shown in Fig. 2. Dispersions of vibration frequencies $\Omega(\mathbf{k})$, calculated in large supercells at $T=0 \mathrm{~K}$, are all positive, except for very long wavelengths as $\lambda \rightarrow \infty$, due to an artifact of numerical calculations. This result is the manifestation of dynamical stability and shows that phonons are unable to mediate any structural transformation. To test whether this structure in a local minimum can resist thermal excitations at high temperatures, we performed $a b$ initio molecular dynamic calculations at finite temperature (AIMD) starting from $0 \mathrm{~K}$ and performing simulations at $300 \mathrm{~K} \rightarrow 600 \mathrm{~K} \rightarrow 900 \mathrm{~K} \rightarrow 1200 \mathrm{~K}$ for 2 ps at each constant temperature step. While the structure has been preserved even at $T=900 \mathrm{~K}$, sizable deformations started at $T=1200 \mathrm{~K}$, but $\mathrm{Si}$ atoms did not dissociate. We also performed annealing tests by reducing the temperature of Si-dohs down to $300 \mathrm{~K}$ within $0.3 \mathrm{ps}$ and then further down to $0 \mathrm{~K}$ within $0.3 \mathrm{ps}$ in Fig. 2. At the end, the deformed Si-dohs structure returned to the original equilibrium structure at $0 \mathrm{~K}$ attained after structure optimization.

All these stringent tests, i.e., structure optimization resulting with a positive cohesive energy close to that of the $\mathrm{Si}-\mathrm{CD}$ as a global minimum, positive frequencies of vibrational modes, structure keeping its identity at high
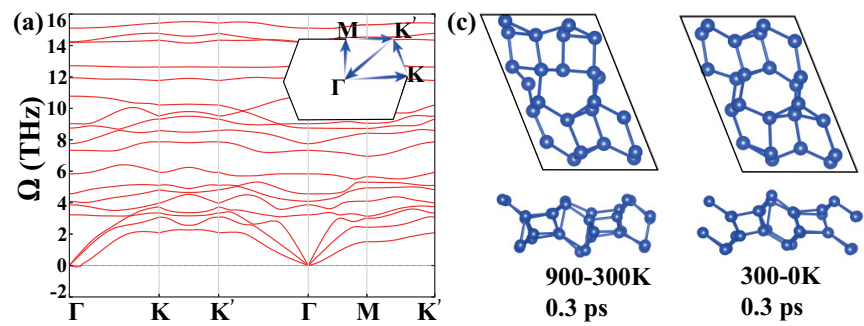
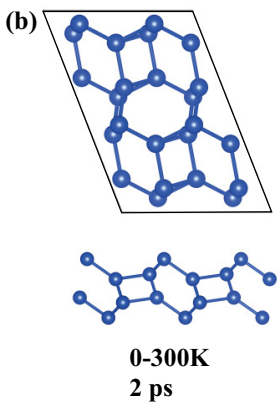

FIG. 2. (a) Variation of frequency $\Omega(\mathbf{k})$ of the vibration modes of Si-dohs structure along high-symmetry directions of Brillouin zone shown by inset. (b) Top and side views of atomic configurations of Si atoms obtained AIMD calculations at high temperature. The temperature has been raised from 0 to $900 \mathrm{~K}$ in three steps with 2-ps simulation time at each step. (c) AIMD calculations for the annealing; $900 \mathrm{~K} \rightarrow 300 \mathrm{~K}$ in $0.3 \mathrm{ps} \rightarrow 0 \mathrm{~K}$ in 0.3 ps.

temperatures and annealing from high temperature back to its equilibrium at $0 \mathrm{~K}$ in AIMD calculations, make us strongly believe that $\mathrm{Si}-d o h s$ is a stable structure corresponding to a local minimum below that of silicene. This stable structure is a semiconductor as demonstrated by the calculated energy-band structure and the total density of states (DOS) in Fig. 3. The

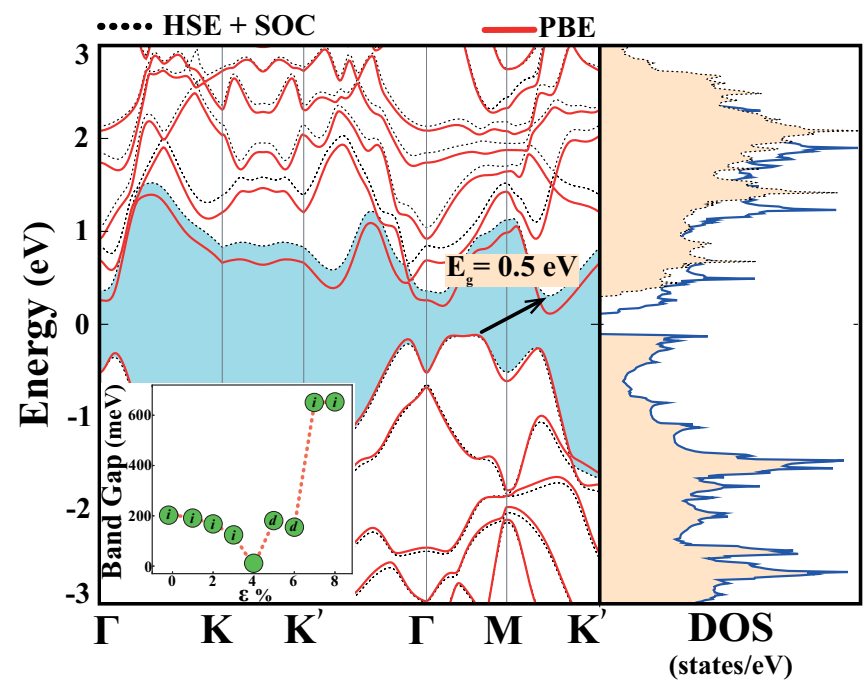

FIG. 3. Electronic energy-band structure of the optimized 2D Si-dohs and corresponding total density of states (DOS) calculated using PBE (solid lines) and the HSE functional including SOC (dotted lines). The band gap between the conduction and valence bands is shaded, and the indirect band gap $E_{g, i}$ is indicated by an arrow. The inset shows the variation of the fundamental band gap with uniaxial strain $(0<\epsilon<8) \%$. 
fundamental band gap of the optimized structure calculated within PBE approximation [30] is indirect and is $E_{g, i}=$ $228 \mathrm{meV}$, whereas the band gap of $E_{g, i}=495 \mathrm{meV}$ is obtained using the HSE functional [32] including the spin orbit coupling (SOC).The fundamental band gap displays interesting and nonmonotonous variation under uniaxial strain as shown by the inset in Fig. 3; it decreases first with increasing $\epsilon$ and eventually the gap is closed at $\epsilon=4 \%$, but the gap reopens for $\epsilon \geqslant 5 \%$ and starts to increase. This occurs since the energies of different bands change differently with strain, and can provide an excellent medium to tune electronic properties.

We next explore whether the bilayer (BL) and 3D periodic (P) structures can be constructed from the vertical stacking of Si-dohs. In Fig. 4(a) are shown three possible stacking sequences and their optimized atomic structures together with their cohesive energies $E_{c}$. These are $\mathrm{AA}^{\prime}, \mathrm{AA}$, and $\mathrm{AB}$ stacking sequences. While BL-AA' and BL-AA transform

\section{Bilayer}
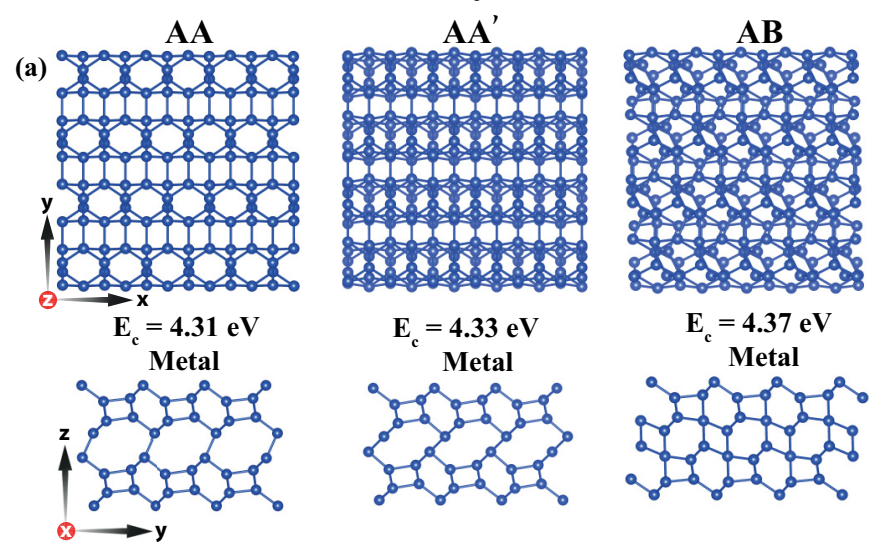

\section{Multilayer (Bulk)}
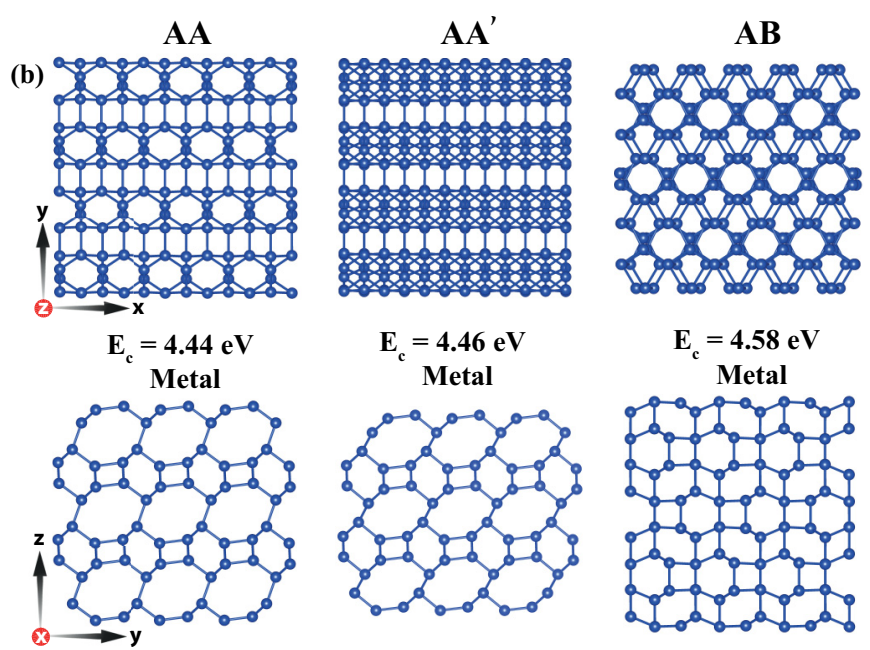

FIG. 4. (a) Top and side views of optimized atomic structure of bilayers (BLs) constructed from three different vertical stacking sequences of $\mathrm{Si}-$ dohs and their cohesive energies $E_{c}$. These are $\mathrm{AA}, \mathrm{AA}^{\prime}$, and $\mathrm{AB}$. BL-AA and BL-AA' transform to $\mathrm{BL}-\mathrm{AB}$ at $T \sim 300 \mathrm{~K}$. (b) Top and side views of the optimized, 3D periodic structures constructed from $\mathrm{AA}, \mathrm{AA}^{\prime}$, and $\mathrm{AB}$ vertical stacking of Si-dohs. to $\mathrm{BL}-\mathrm{AB}$ at $T \sim 300 \mathrm{~K}, \mathrm{BL}-\mathrm{AB}$ is able to maintain their structure at $T=900 \mathrm{~K}$, without dissociation. BL-AB is a metal and has the cohesive energy, $E_{c}=4.37 \mathrm{eV} /$ atom. Since a BL of Si-dohs can remain stable, 3D periodic structures may be constructed with vertical $\mathrm{AA}^{\prime}, \mathrm{AB}$, and AA stacking. The optimized, 3D periodic structures are described in Fig. 4(b). Further to 3D CD-Si, wurtzite-Si and other phases obtained in extreme conditions in diamond anvil, the present study deduces that there are three different, metallic 3D structures of Si. Incidentally, these three structures are different from 3D silicon allotropes [43-45]. Furthermore, they have dynamical stability at $T=0 \mathrm{~K}$ and can remain stable at $900 \mathrm{~K}$. P-AA

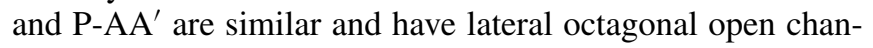
nels, so that their quasilayered structure becomes discernible. Nonetheless, these 3D structures are not like layered graphite, that is constructed by the stacking of graphene monolayers bound to adjacent layers by weak van der Waals interaction, but they rather have low-density vertical bonds [26] between layers. As for the $\mathrm{P}-\mathrm{AB}$ structure, it has cohesion slightly higher than those of P-AA and P-AA' and does not display a layered character. The formation of strong and vertical covalent $\mathrm{Si}$-Si bonds destroys the fully layered nature. In this respect, these 3D Si phases are similar to silicite constructed from trigonal and honeycomb dumbbell layers [15].

Here the crucial question to be addressed is whether interesting structural and electronic properties unveiled for free-standing $\mathrm{Si}-d o h s$ can be maintained when it is placed (or grown) on specific substrates for specific applications. We answered this question by studying $\mathrm{Si}-d o h s$ supported by $\operatorname{Ag}(111)$ and graphene substrates. To ensure the lattice matching for the overlayer and substrate within periodically repeating supercells, $\operatorname{Ag}(111)$ and graphene were slightly strained and the structure of Si-dohs, which was placed on the substrates at large spacing, has been reoptimized. It should be noted that under strain these substrates usually become more reactive to interact more strongly with the overlayer. On the contrary, the interaction between incommensurate overlayer and substrate can be weakened. In this respect, strained substrates may constitute stringent tests for possible substrate effects on Si-dohs overlayers. Top and side views of optimized atomic structures of Si-dohs on $\mathrm{Ag}(111)$ in Fig. 5(a) indicate that the overlayer approaches and attains a small spacing to set a strong coupling with the substrate, so that the semiconducting character of the overlayer changed to metallic. The charge density isosurfaces corroborate this conclusion. In contrast to $\mathrm{Ag}(111)$ substrate, $\mathrm{Si}-d o h s$ becomes bound to graphene at a large spacing via a weak van der Waals interaction, so that its semiconducting character is maintained as shown in Fig. 5(b).

This allotrope can be chemically doped by adatoms to modify its electronic structure locally and to create localized acceptor and donor states near the edges of conduction- and valence-band edges as shown in Fig. 6. The chemical doping is simulated by the adsorption of a single adatom to each $6 \times 4$ supercell of Si-dohs, whereby the adsorbate-absorbate interaction is minimized. Our analysis indicates that isocoric adatoms like single $\mathrm{Al}$ and $\mathrm{P}$ are adsorbed on $\mathrm{Si}-d o h s$ with strong binding energy of 3.0 and $3.6 \mathrm{eV}$, respectively. These adatoms, also used in the doping of CD-Si, construct empty and filled localized states at the edges of the fundamental band 


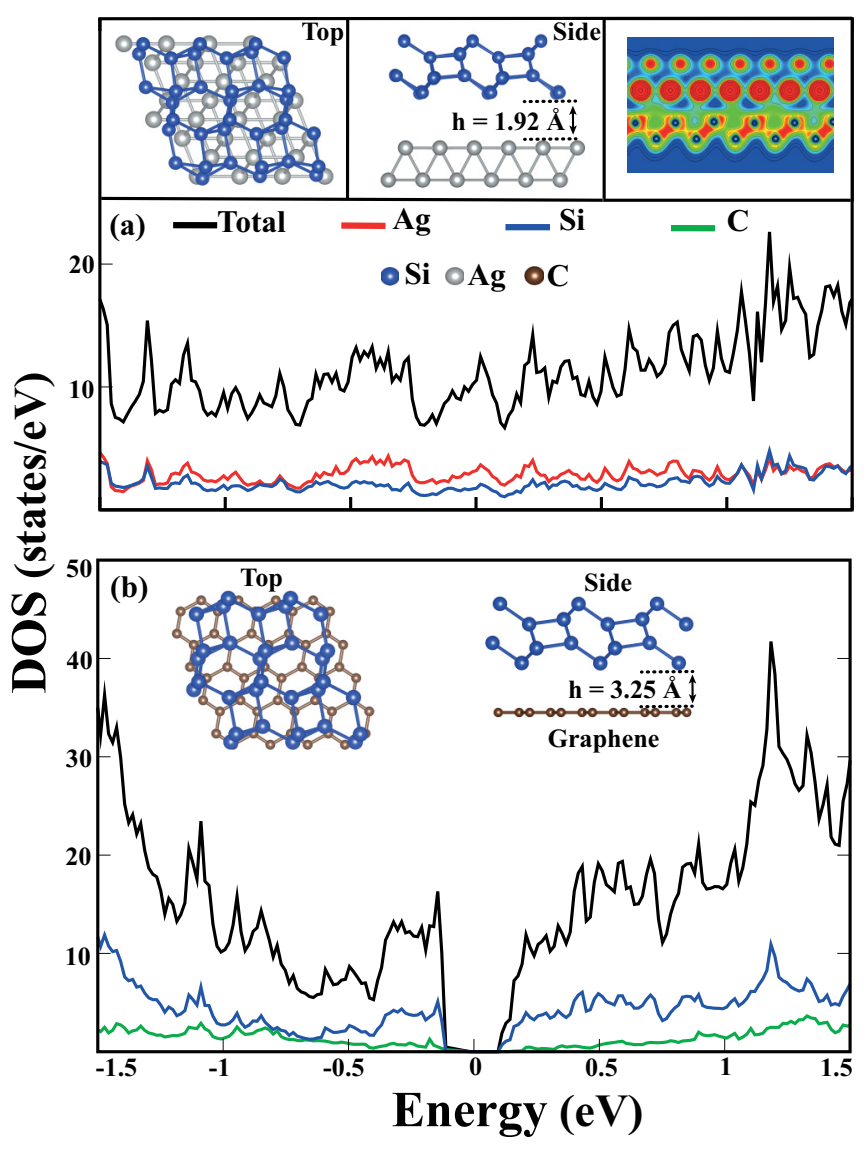

FIG. 5. Substrate effects on Si-dohs. (a) Top and side views of optimized atomic structure of $\mathrm{Si}$-dohs overlayer on $\mathrm{Ag}(111)$ substrate. The total density of states (DOS) and those projected (PDOS) to $\mathrm{Si}$ and $\mathrm{Ag}$ atoms display a metallic character. Strong overlayer-substrate interaction is clarified by the charge density contour plots shown by inset. (b) Same for Si-dohs on graphene with a large spacing between them. DOS and PDOS indicate that the semimetallic state of the substrate and semiconductor state of the Si-dohs overlayer are preserved.

gap of Si-dohs, which is deduced by projecting the density of states to the host Si atom far from the adsorbate.

Here, we briefly discuss another important property of Sidohs, which is functionalized (or decorated) by $3 d$ transition metal atoms, $\mathrm{Cr}, \mathrm{V}$, and $\mathrm{Ti}$ to form quasi-2D clathrates as shown in Fig. 7(a). These clathrate structures, where the transition metal atoms are kept in the hexagonal rings, are dynamically and thermally stable. In Fig. 7(b), the Cr-decorated Si-dohs, namely $2 \mathrm{D} \mathrm{CrSi}_{6}$, attains a permanent magnetic moment of $\sim 2.0 \mu_{B}$ with a spin polarized half-metallic state. Hence, the HSE bands is metallic for spin-up states, but semiconducting for the spin-down states with a band gap of $0.88 \mathrm{eV}$. Accordingly, $\mathrm{CrSi}_{6}$ can operate as a spin valve for spin-down states. In Fig. 7(c), the HSE bands calculated for a structure optimized $\mathrm{VSi}_{6}$ clathrate depict a magnetic semiconductor with the band gap of $0.20 \mathrm{eV}$ for spin-up states, but a relatively larger band gap of $0.83 \mathrm{eV}$ for spindown states. As for $\mathrm{TiSi}_{6}$, it is a nonmagnetic semiconductor with an indirect band gap of $0.4 \mathrm{eV}$ obtained using the HSE functional. (a)
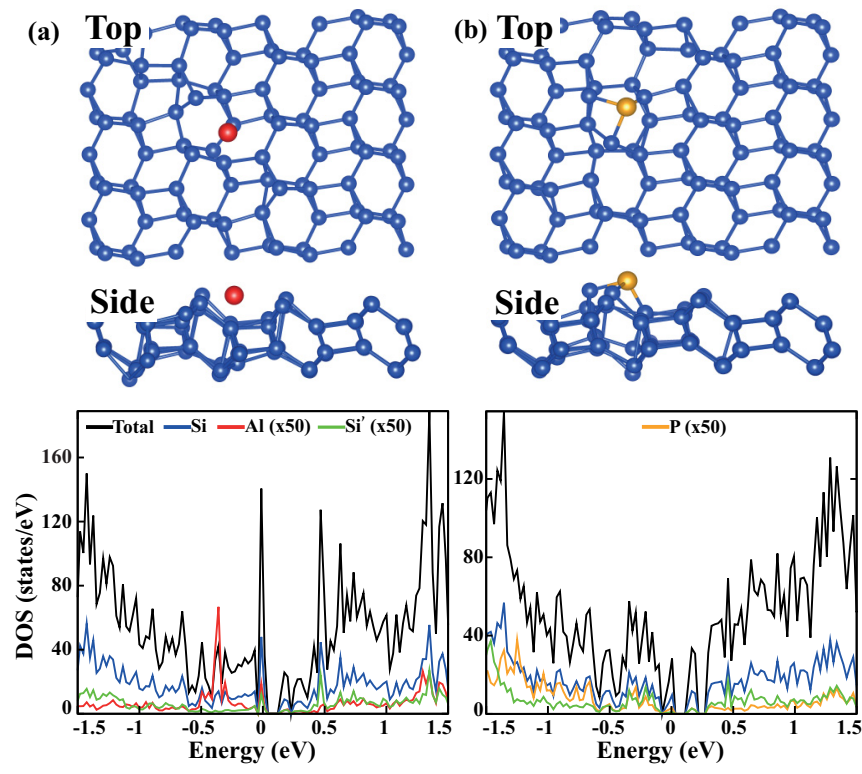

FIG. 6. (a) Top and side views of equilibrium atomic structure of the $\mathrm{Si}$-dohs phase after the adsorption of $\mathrm{Al}$ atom and calculated the total and adatom projected densities of states for a single $\mathrm{Al}$ adatom adsorbed to $6 \times 4$ supercell of $\mathrm{Si}$-dohs phase. (b) Top and side views of the atomic configuration of $6 \times 4$ supercell of $\mathrm{Si}$-dohs used to tread the adsorbed $\mathrm{P}$ adatom and the total and adatom projected densities of states calculated for a single $P$ adatom adsorbed to $6 \times 4$ supercell of Si-dohs phase at equilibrium sites.

\section{OTHER GROUP-IV AND GROUP-V ELEMENTS AND III-V COMPOUND}

Earlier, 3D crystals of group IV elements and their binary compounds, as well as group III-V compounds, which are well known in materials science and device technology with their optoelectronic properties, were found to construct 2D stable, free-standing planar or buckled honeycomb structures [2,3,7]. Some of these elements and compounds, such as C, Ge, AlP, and GaAs, are also found to form stable, 2D allotropes like Si-dohs as shown in Fig. 8. Even though their band gaps are underestimated by PBE calculations, all these structures,

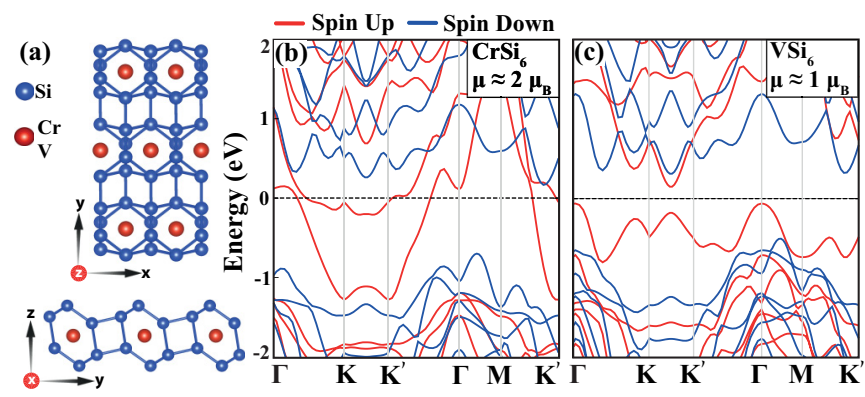

FIG. 7. Si-dohs decorated by transition metal atoms forming a quasi-2D clathrate structure, where $\mathrm{Cr}$ or $\mathrm{V}$ atoms are kept in the hexagonal rings. (a) Quasi-2D $\mathrm{CrSi}_{6}$ or $\mathrm{VSi}_{6}$ clathrate structures: Top and side views of optimized atomic structure. (b) Spin-split energy bands calculated by HSE. (c) Same as (b) for quasi-2D VSi ${ }_{6}$ clathrate. The zero energy is set to the Fermi level. Spin-up and spin-down bands are highlighted by different colors. 

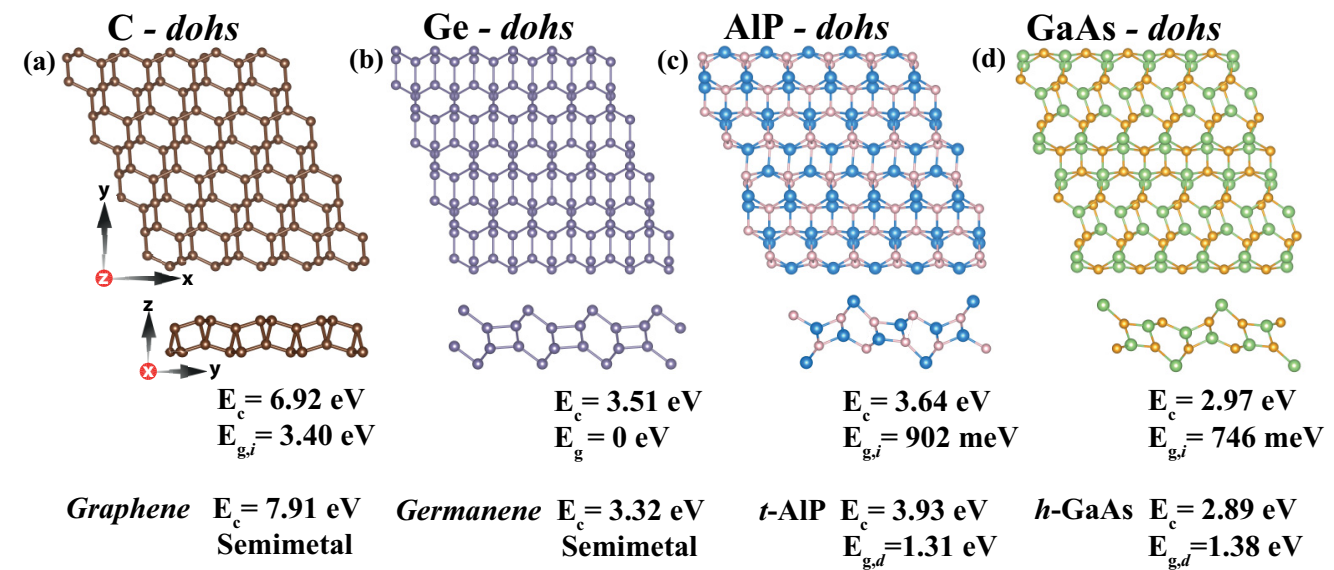

Germanene $\begin{array}{r}\mathrm{E}_{\mathrm{c}}=3.32 \mathrm{eV} \\ \text { Semimetal }\end{array}$

$$
\begin{array}{r}
t-\mathrm{AIP} \\
\mathrm{E}_{\mathrm{c}}=3.93 \mathrm{eV} \\
\mathrm{E}_{\mathrm{g} d}=1.31 \mathrm{eV}
\end{array}
$$$$
h \text {-GaAs } \begin{array}{r}
\mathrm{E}_{\mathrm{c}}=2.89 \mathrm{eV} \\
\mathrm{E}_{\mathrm{o} d}=1.38 \mathrm{eV}
\end{array}
$$

FIG. 8. Top and side views of optimized atomic structure of some other group-IV elements $\mathrm{C}$, Ge, and group-III-V compounds GaAs, AlP constructing stable 2D structures like Si-dohs. The PBE calculated (average) cohesive energy $E_{c}$ per atom and fundamental band gap is presented. For comparison, values calculated for counterparts in 2D hexagonal structure are also given.

in particular Ge-dohs, are semiconductors with fundamental band gaps smaller than their bulk counterparts in diamond or zinc-blende structures. The structure of C-dohs differs slightly from that of Si-dohs, perhaps, due to strong $\pi-\pi$ orbital interaction at smaller bond distance. We note that AlP-dohs and $\mathrm{Si}$-dohs having similar structure and lattice constants are suitable to form vertical heterostructures offering different device capabilities.

Surprisingly, not only group-IV elements and III-V compounds, but also phosphorous $\mathrm{P}$ being a group- $\mathrm{V}$ element, construct 2D stable, monolayer dohs structure. The dynamical and thermal stability of P-dohs are ensured through phonon and finite-temperature $a b$ initio molecular dynamics (AIMD) calculations. This structure maintained its stability up to $900 \mathrm{~K}$. Moreover, it preserved its stability by keeping the original structure after the annealing from 900 to $300 \mathrm{~K}$ within $0.3 \mathrm{ps}$ and from 300 to $0 \mathrm{~K}$ within $0.3 \mathrm{ps}$. The $\mathrm{P}$-dohs structure exhibits a semimetalic band in contrast to other well-known allotropes, such as blue and black phosphorene monolayers [46-48]. Valance and conducting bands have a very small overlap near the Fermi level with the PBE and HSE functional. Unlike the Si-dohs structure, P-dohs has more deformed octagons and proper hexagons [Fig. 9(a)] like the $3 \mathrm{D} \mathrm{K}_{4}$ phosphorus structure [49]. The P-dohs structure has less cohesive energy compared with black and blue phosphorene.

\section{DISCUSSION AND CONCLUSION}

The predictions of the present study on the stable, 2D silicon structure with high cohesion differ from the $2 \mathrm{D} \mathrm{Si}$ structures discussed above in the following aspects, and make an important contribution to the field: (i) The atomic structure of the present allotrope does not have any bearing on the those discussed above; it has deformed eight-, six-, and fourmembered rings in the same structures. It is rather robust; it has dynamical stability and thermal stability at high temperature. (ii) This allotrope is not a computer generated structure. We showed that the dohs structure can be driven from the quasilayered $\mathrm{P} 6 / \mathrm{m}-\mathrm{Si}_{6}$ structure. Therefore, a kinetic path to generate the dohs structure is known. (iii) Si-dohs is one of the 2D allotropes of $\mathrm{Si}$, which has highest cohesion (or lowest energy per atom) within the numerical uncertainty of DFT calculations depending on the calculational parameters. (iv) It is an indirect band gap semiconductor with a $0.5 \mathrm{eV}$ band gap. This band gap is closed for uniaxial tensile strain of $4 \%$, but it reappears and increases with further increase of the strain offering an excellent tunability. (v) We showed that single-layer Si-dohs can form stable, but metallic, bilayers.

(a)
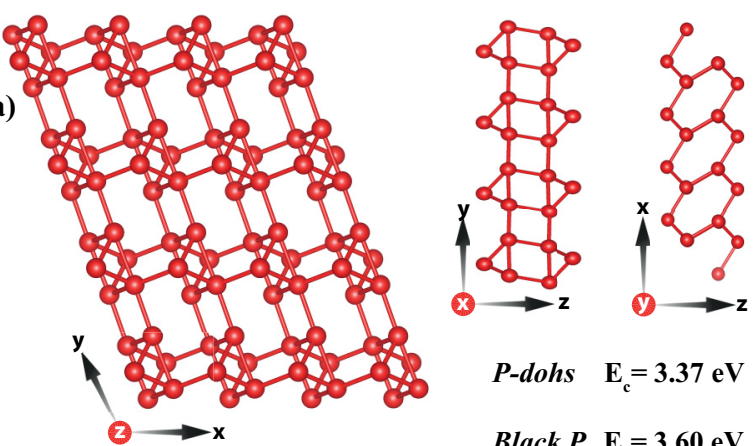

Black P. $\mathrm{E}_{\mathrm{c}}=3.60 \mathrm{eV}$ Blue P. $\quad \mathrm{E}_{\mathrm{c}}=3.57 \mathrm{eV}$

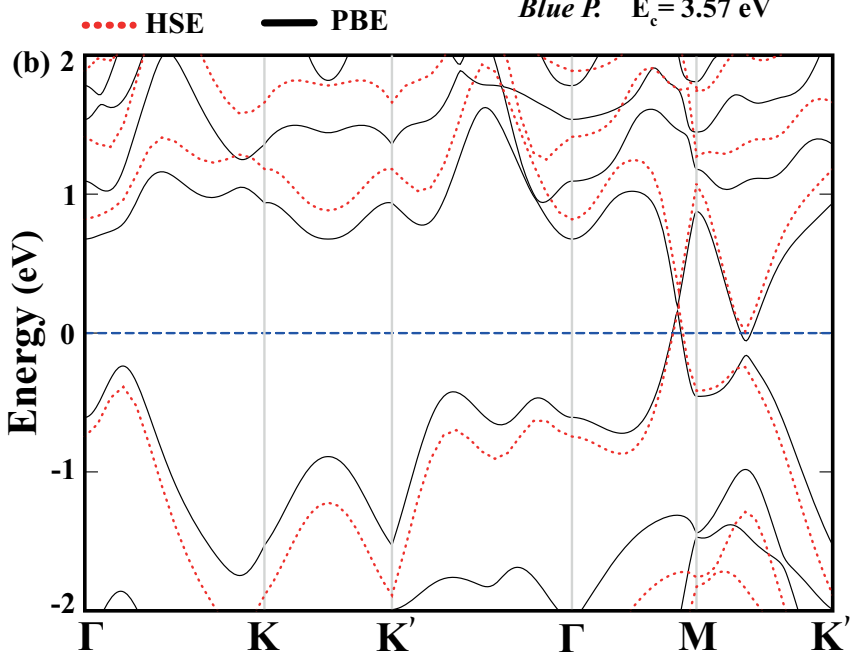

FIG. 9. (a) Top and side views of optimized atomic structure of P-dohs. (b) Energy bands structure calculated using PBE (solid lines) and HSE (dotted lines) functional. 
Furthermore, we showed that one can construct three metallic phases of 3D Si through vertical stacking of monolayer Sidohs in different registries. We believe that this is an important prediction towards the realization of 3D metallic Si crystal. (vi) We also showed that monolayer Si-dohs keeps its identity and electronic properties to a great extent when placed on graphene. However, its semiconducting properties cease when it grows on a metal surface like $\mathrm{Ag}(111)$. (vii) Si-dohs can easily be doped by isocoric impurities like $\mathrm{Al}$ and $\mathrm{P}$ atoms to have acceptor and donor states. Additionally, we showed that Sidohs can form 2D clathrate structures with $3 d$ transition metal atoms. $\mathrm{CrSi}_{6}$ clathrate is a half metal with a band gap of $0.9 \mathrm{eV}$ for spin-down states, whereas $\mathrm{VSi}_{6}$ is a magnetic semiconductor with two different band gaps for different spin polarization. On the other hand, $\mathrm{TiSi}_{6}$ is a narrow band-gap semiconductor. (viii) Finally, the dohs structure appears to be a stable structure, not only for $\mathrm{Si}$, but also for other group-IV elements ( $\mathrm{C}$ and $\mathrm{Ge}$ ), group-III-V compounds (GaAs, AlP), and also a group- $\mathrm{V}$ element $(\mathrm{P})$. In this respect, the dohs structure constitutes a large class of materials like the honeycomb structure. Perhaps this is the most important aspect of this paper.

In conclusion, starting from the metallic $3 \mathrm{D} \mathrm{P} 6 / \mathrm{m}_{-} \mathrm{Si}_{6}$ clathrate of silicon we found a $2 \mathrm{D}$, single-layer allotrope of $\mathrm{Si}$ specified as $\mathrm{Si}-d o h s$, which is dynamically stable and can resist high-temperature excitation. In contrary to the wellknown semimetallic allotrope, silicene, it is a semiconductor with an indirect fundamental band gap and has relatively stronger cohesion. This single-layer allotrope can form metallic bilayers and even periodic, 3D-layered-like structures. Additionally, when supported by specific substrates, this allotrope can maintain its properties calculated for its free-standing state. We showed that this allotrope can be chemically doped by adatoms like $\mathrm{Al}$ and $\mathrm{P}$ to generate acceptor and donor states. It can be functionalized to construct stable, quasi-2D clathrate structures with light transition metal atoms and acquires magnetic properties, like a half-metallic state. Most interestingly, not only $\mathrm{Si}$, but also some other group-IV elements, as well as group-III-V compounds, can have allotropes like Si-dohs. Surprisingly, even phosphorus, a group-V element, can form a stable dohs structure. In contrast to black and blue phosphorene, P-dohs is a semimetal. In contrast to PBE bands, the HSE yields a band inversion leading to a nontrivial topological state. We note that AlPdohs and Si-dohs having similar structure and lattice constants are suitable to form vertical heterostructures offering interesting device capabilities. Similarly, a junction of Si-dohs/Pdohs can generate an interesting Schottky diode. All these predictions point to the fact that these $2 \mathrm{D}$ allotropes revealed in the present study can be suitable for applications in $2 \mathrm{D}$ nanoelectronics/spintronics and herald an interesting class of nanomaterials.

\section{ACKNOWLEDGMENTS}

The computational resources were provided by TÜBITAK ULAKBIM, High Performance and Grid Computing Center (TR-Grid e-Infrastructure). This research was supported by the TÜBITAK under Project No. 116F059. S.C. acknowledges financial support from the Academy of Sciences of Turkey TÜBA.
[1] K. S. Novoselov, A. Geim, S. Morozov, D. Jiang, Y. Zhang, S. Dubonos, I. Grigorieva, and A. Firsov, Science 306, 666 (2004).

[2] E. Durgun, S. Tongay, and S. Ciraci, Phys. Rev. B 72, 075420 (2005).

[3] S. Cahangirov, M. Topsakal, E. Akturk, H. Sahin, and S. Ciraci, Phys. Rev. Lett. 102, 236804 (2009).

[4] S. Cahangirov, M. Topsakal, and S. Ciraci, Phys. Rev. B 81, 195120 (2010).

[5] K. S. Novoselov, D. Jiang, F. Shedin, T. Booth, V. V. Khotkevich, S. Morozov, and A. K. Geim, Proc. Natl. Acad. Sci. USA 102, 10451 (2005).

[6] M. Topsakal, E. Akturk, and S. Ciraci, Phys. Rev. B 79, 115442 (2009)

[7] H. Sahin, S. Cahangirov, M. Topsakal, E. Bekaroglu, E. Akturk, R. T. Senger, and S. Ciraci, Phys. Rev. B 80, 155453 (2009).

[8] M. Topsakal, S. Cahangirov, E. Bekaroglu, and S. Ciraci, Phys. Rev. B 80, 235119 (2009).

[9] P. Vogt, P. DePadova, C. Quaresima, J. Avila, E. Frantzeskakis, M. C. Asensio, A. Resta, B. Ealet, and G. LeLay, Phys. Rev. Lett. 108, 155501 (2012).

[10] B. Feng, Z. Ding, S. Meng, Y. Yao, X. He, P. Cheng, L. Chen, and K. Wu, Nano Lett. 12, 3507 (2012).

[11] P. D. Padova, P. Vogt, A. Resta, J. Avila et al., Appl. Phys. Lett. 102, 163106 (2013).

[12] S. Cahangirov, V. O. Özçelik, L. Xian, J. Avila, S. Cho, M. C. Asensio, S. Ciraci, and A. Rubio, Phys. Rev. B 90, 035448 (2014).
[13] P. D. Padova, C. Ottaviani, C. Quaresima, B. Oliviera et al., 2D Mater. 1, 021003 (2014).

[14] L. Chen, C.-C. Liu, B. Feng, X. He, P. Cheng, Z. Ding, S. Meng, Y. Yao, and K. Wu, Phys. Rev. Lett. 109, 056804 (2012).

[15] S. Cahangirov, V. O. Ozcelik, A. Rubio, and S. Ciraci, Phys. Rev. B 90, 085426 (2014).

[16] V. O. Ozcelik, E. Durgun, and S. Ciraci, J. Phys. Chem. Lett. 5, 2694 (2014).

[17] E. Aktürk, C. Ataca, and S. Ciraci, Appl. Phys. Lett. 96, 123112 (2010).

[18] Z. Zhuo, X. Wu, and J. Yang, Nanoscale 10, 1265 (2018).

[19] P. Borlido, C. Rödl, M. A. L. Marques, and S. Botti, 2D Mater. 5, 035010 (2018).

[20] K. Chae, D. Y. Kim, and Y.-W. Son, 2D Mater. 5, 025013 (2018).

[21] R. Yaokawa, T. Ohsuna, T. Morishita, Y. Hayasaka, M. J. S. Spencer, Nat. Commun. 7, 10657 (2105).

[22] Y. Sakai and A. Oshiyama, Phys. Rev. B 91, 201405 (2015).

[23] W. Luo, Y. Ma, X. Gong, and H. Xiang, J. Am. Chem. Soc. 136, 15992 (2014).

[24] T. Morishita, M. J. S. Spencer, S. Russo, I. K. Snook, and M. Mikami, Chem. Phys. Lett. 506, 221 (2011).

[25] M. A. Zwijnenburg, K. E. Jelfs, and S. T. Bromley, Phys. Chem. Chem. Phys. 12, 8505 (2010).

[26] A. Onen, D. Kecik, E. Durgun, and S. Ciraci, Nanoscale 10, 21842 (2018). 
[27] P. E. Blöchl, Phys. Rev. B 50, 17953 (1994).

[28] G. Kresse and J. Furthmüller, Phys. Rev. B 54, 11169 (1996).

[29] G. Kresse and J. Furthmüller, Comput. Mater. Sci. 6, 15 (1996).

[30] J. P. Perdew, K. Burke, and M. Ernzerhof, Phys. Rev. Lett. 77, 3865 (1996).

[31] S. Grimme, J. Comput. Chem. 27, 1787 (2006).

[32] J. Heyd, G. E. Scuseria, and M. Ernzerhof, J. Chem. Phys. 118, 8207 (2003).

[33] A. Togo and I. Tanaka, Scr. Mater. 108, 1 (2015).

[34] H.-J. Sung, W. H. Han, I.-H. Lee, K. J. Chang, Phys. Rev. Lett. 120, 157001 (2018).

[35] M. Hanfland, U. Schwarz, K. Syassen, and K. Takemura, Phys. Rev. Lett. 82, 1197 (1999).

[36] O. O. Kurakevych, T. A. Strobel, D. Y. Kim, T. Muramatsu, and V. V. Struzhkin, Cryst. Growth Des. 13, 303 (2013).

[37] L. Rapp, B. Habel, C. J. Pickard, J. E. Bradby, E. G. Gamaly, J. S. Williams, and A. V. Rode, Nat. Commun. 6, 755 (2015).

[38] T. Gorkan, İ. Ozdemir, M. Y. Bakır, F. Ersan, G. Gökoğlu, E. Aktürk, and S. Ciraci, J. Phys. Chem. C. 123, 24 (2019).
[39] D. Liu and D. Tomanek, Nano Lett. 19, 1359 (2019).

[40] H. Fu, J. Zhang, Z. Ding, H. Li, and S. Meng, Appl. Phys. Lett. 104, 131904 (2014).

[41] J. E. Padiha and R. B. Pontes, J. Phys. Chem. C 119, 3818 (2015).

[42] Y. Aierken and O. Leenaerts, F. M. Peeters, Phys. Chem. Chem. Phys. 18, 18486 (2016).

[43] D. Y. Kim, S. Stefanoski, O. O. Kurakevych, and T. A. Strobel, Nat. Mater. 14, 169 (2015).

[44] G. B. Adams, M. OKeeffe, A. A. Demkov, O. F. Sankey, and Y.-M. Huang, Phys. Rev. B 49, 8048 (1994).

[45] M. Amsler, S. Botti, M. A. L. Marques, T. J. Lenosky, and S. Goedecker, Phys. Rev. B 92, 014101 (2015).

[46] J. Guan, Z. Zhu, and D. Tománek, Phys. Rev. Lett. 113, 046804 (2014).

[47] Z. Zhu and D. Tomanek, Phys. Rev. Lett. 112, 176802 (2014).

[48] H. Liu, A. T. Neal, Z. Zhu, Z. Luo, X. Xu, D. Tomanek, and P. D. Ye, ACS Nano 8, 4033 (2014).

[49] J. Liu, S. Zhang, Y. Guo, and Q. Wang, Sci. Rep. 6, 37528 (2016). 